\title{
Determining the Higgs spin and parity in the diphoton decay channel
}

\author{
Daniël Boer, ${ }^{1}$ Wilco J. den Dunnen, ${ }^{2}$ Cristian Pisano, ${ }^{3}$ and Marc Schlegel $^{2}$ \\ ${ }^{1}$ Theory Group, KVI, University of Groningen, Zernikelaan 25, NL-9747 AA Groningen, The Netherlands \\ ${ }^{2}$ Institute for Theoretical Physics, Universität Tübingen, \\ Auf der Morgenstelle 14, D-72076 Tübingen, Germany \\ ${ }^{3}$ Dipartimento di Fisica, Università di Cagliari, and INFN, \\ Sezione di Cagliari, I-09042 Monserrato (CA), Italy
}

(Dated: December 3, 2018)

\begin{abstract}
We calculate the diphoton distribution in the decay of arbitrary spin- 0 and spin- 2 bosons produced from gluon fusion, taking into account the fact that gluons inside an unpolarized proton are generally linearly polarized. The gluon polarization brings about a difference in the transverse momentum distribution of positive and negative parity states. At the same time, it causes the azimuthal distribution of the photon pair to be non-isotropic for several spin-2 coupling hypotheses, allowing one to distinguish these from the isotropic scalar and pseudoscalar distributions.
\end{abstract}

PACS numbers: 12.38.-t; 13.85.Ni; $13.88 .+\mathrm{e}$

Last year July it was announced that a new boson with a mass around $125-126 \mathrm{GeV}$ was observed by both the ATLAS [1] and CMS [2] collaborations. An excess of events was observed in $\gamma \gamma, Z Z^{*}$ and $W W^{*}$ production from proton-proton collisions at a center of mass energy of 7 and $8 \mathrm{TeV}$. The observed excess is consistent, within uncertainties, with the production and decay of the Standard Model (SM) Higgs boson.

Now that the existence of a new particle has been established, both collaborations have begun the determination of its spin and parity. Both ATLAS [3-5] and CMS $[6,7]$ set approximately $3 \sigma$ exclusions on the $J^{P}=0^{-}$ scenario using the $Z Z^{*}$ channel and the $2_{m}^{+}$hypothesis ${ }^{1}$ starts to be disfavored at the 1-3 $\sigma$ level in the $\gamma \gamma, Z Z^{*}$ and $W W^{*}$ channels. As the decay of a pure spin- 1 state to two photons is not allowed according to the LandauYang theorem $[8,9]$, the $\gamma \gamma$ channel is being used to distinguish between spin-0 and spin-2 only. In the $Z Z^{*}$ and $W W^{*}$ decay channels, the spin-1 option should also be considered.

Even though the number of events is much larger in the $\gamma \gamma$ channel, the ability to distinguish spin- 0 from spin-2 is not much better than in the $Z Z^{*}$ channel. The reason is that in the $\gamma \gamma$ channel only the distribution of the polar angle $\theta$ is considered $[3,10]$. The spin- 0 and spin-2 hypotheses are not very different in this variable after experimental acceptance cuts [3], leading to a small discriminating power. The determination of the parity using only this angle is even impossible, as the distributions of $0^{+}$and $0^{-}$are exactly equal and the same holds true for the $2_{h}^{ \pm}$scenarios ${ }^{2}$.

\footnotetext{
1 The coupling of a spin-2 boson to gauge bosons can be realized in multiple ways. We will use the standard notation in which $2_{m}^{+}$denotes a spin-2 boson with minimal (lowest dimensional) coupling, which is uniquely defined.

2 The $h$ subscript indicates that the spin- 2 boson couples through
}

In this letter we demonstrate that one can also differentiate between the different spin scenarios in the $\gamma \gamma$ channel, by studying the dependence on the azimuthal angle $\phi$ in the Collins-Soper frame [13], which is the diphoton restframe with the $\hat{x} \hat{z}$-plane spanned by the 3 -momenta of the colliding protons and the $\hat{x}$-axis set by their bisector. Moreover, different spin-2 coupling hypotheses that have an equal $\theta$ dependence can be distinguished from each other using the $\phi$ distribution, enhancing the analyzing potential of this channel. Apart from that, we update predictions for the transverse momentum distribution $[14,15]$ which can, in principle, be used to distinguish the different parity states $0^{+}$from $0^{-}$and $2_{h}^{+}$ from $2_{h}^{-}$in the $\gamma \gamma$ channel. Azimuthal angular distributions have been discussed for spin-0 and spin-2 "Higgs" production from vector-boson fusion [16-18], but not yet from gluon fusion and not including linear polarization.

A non-trivial $\phi$ distribution in the decay of spin-2 bosons produced from gluon fusion can be caused by the fact that gluons in an unpolarized proton are generally linearly polarized. The degree of gluon polarization can be calculated using perturbative QCD (pQCD) for transverse momentum of the gluon much larger than the proton mass and is found to be large. For small transverse momentum pQCD cannot be used to calculate the degree of polarization, but this lack of knowledge turns out to be of little influence on the final $\phi$ distribution, which is mostly dominated by the perturbative part.

The effects of gluon polarization can be described in the framework of Transverse Momentum Dependent (TMD) factorization. In that framework, the full $p p \rightarrow$ $\gamma \gamma X$ cross section is split into a partonic $g g \rightarrow \gamma \gamma$ cross section and two TMD gluon correlators, which describe

a higher-dimensional coupling. There are multiple higherdimensional couplings possible. We follow the convention of Ref. $[11,12]$ for $2_{h}^{ \pm}$. 
the distribution of gluons inside a proton as a function of not only its momentum along the direction of the proton, but also transverse to it. More specifically, the differential cross section for the inclusive production of a photon pair from gluon-gluon fusion is written as [19, 20],

$$
\begin{array}{r}
\frac{\mathrm{d} \sigma}{\mathrm{d}^{4} q \mathrm{~d} \Omega} \propto \int \mathrm{d}^{2} \boldsymbol{p}_{T} \mathrm{~d}^{2} \boldsymbol{k}_{T} \delta^{2}\left(\boldsymbol{p}_{T}+\boldsymbol{k}_{T}-\boldsymbol{q}_{T}\right) \mathcal{M}_{\mu \rho \kappa \lambda}\left(\mathcal{M}_{\nu \sigma}{ }^{\kappa \lambda}\right)^{*} \\
\Phi_{g}^{\mu \nu}\left(x_{1}, \boldsymbol{p}_{T}, \zeta_{1}, \mu\right) \Phi_{g}^{\rho \sigma}\left(x_{2}, \boldsymbol{k}_{T}, \zeta_{2}, \mu\right),
\end{array}
$$

with the longitudinal momentum fractions $x_{1}=$ $q \cdot P_{2} / P_{1} \cdot P_{2}$ and $x_{2}=q \cdot P_{1} / P_{1} \cdot P_{2}, q$ the momentum of the photon pair, $\mathcal{M}$ the $g g \rightarrow \gamma \gamma$ partonic hard scattering matrix element and $\Phi$ the following unpolarized proton gluon TMD correlator,

$$
\begin{gathered}
\Phi_{g}^{\mu \nu}\left(x, \boldsymbol{p}_{T}, \zeta, \mu\right) \equiv 2 \int \frac{\mathrm{d}(\xi \cdot P) \mathrm{d}^{2} \xi_{T}}{(x P \cdot n)^{2}(2 \pi)^{3}} e^{i\left(x P+p_{T}\right) \cdot \xi} \\
\operatorname{Tr}_{c}\left[\left\langle P\left|F^{n \nu}(0) \mathcal{U}_{[0, \xi]}^{n[-]} F^{n \mu}(\xi) \mathcal{U}_{[\xi, 0]}^{n[-]}\right| P\right\rangle\right]_{\xi \cdot P^{\prime}=0} \\
=-\frac{1}{2 x}\left\{g_{T}^{\mu \nu} f_{1}^{g}-\left(\frac{p_{T}^{\mu} p_{T}^{\nu}}{M_{p}^{2}}+g_{T}^{\mu \nu} \frac{\boldsymbol{p}_{T}^{2}}{2 M_{p}^{2}}\right) h_{1}^{\perp g}\right\}+\mathrm{HT}, \quad(2)
\end{gathered}
$$

with $p_{T}^{2}=-\boldsymbol{p}_{T}^{2}$ and $g_{T}^{\mu \nu}=g^{\mu \nu}-P^{\mu} P^{\prime \nu} / P \cdot P^{\prime}-$ $P^{\prime \mu} P^{\nu} / P \cdot P^{\prime}$, where $P$ and $P^{\prime}$ are the momenta of the colliding protons and $M_{p}$ their mass. The gauge link $\mathcal{U}_{[0, \xi]}^{n[-]}$ in the matrix element runs from 0 to $\xi$ via minus infinity along the direction $n$, which is a time-like dimensionless four-vector with no transverse components such that $\zeta^{2}=(2 n \cdot P)^{2} / n^{2}$. In principle, Eqs. (1) and (2) also contain soft factors, but with the appropriate choice of $\zeta$ (of around 1.5 times the hadronic center of mass energy), one can neglect their contribution, at least up to next-to-leading order [20, 21]. The renormalization scale should be chosen around the characteristic scale of the hard interaction. The last line of Eq. (2) contains the parameterization of the TMD correlator in terms of the unpolarized gluon distribution $f_{1}^{g}\left(x, \boldsymbol{p}_{T}^{2}, \zeta, \mu\right)$, the linearly polarized gluon distribution $h_{1}^{\perp g}\left(x, \boldsymbol{p}_{T}^{2}, \zeta, \mu\right)$ and Higher Twist (HT) terms, which only give $\mathcal{O}(1 / Q)$ suppressed contributions to the cross section, where $Q \equiv \sqrt{q^{2}}$.

The general structure of the differential cross section for the process $p p \rightarrow \gamma \gamma X$ is given by [22]

$$
\begin{aligned}
& \frac{\mathrm{d} \sigma}{\mathrm{d}^{4} q \mathrm{~d} \Omega} \propto F_{1}(Q, \theta) \mathcal{C}\left[f_{1}^{g} f_{1}^{g}\right]+F_{2}(Q, \theta) \mathcal{C}\left[w_{2} h_{1}^{\perp g} h_{1}^{\perp g}\right] \\
& +F_{3}(Q, \theta) \mathcal{C}\left[w_{3} f_{1}^{g} h_{1}^{\perp g}+\left(x_{1} \leftrightarrow x_{2}\right)\right] \cos (2 \phi) \\
& +F_{3}^{\prime}(Q, \theta) \mathcal{C}\left[w_{3} f_{1}^{g} h_{1}^{\perp g}-\left(x_{1} \leftrightarrow x_{2}\right)\right] \sin (2 \phi) \\
& \quad+F_{4}(Q, \theta) \mathcal{C}\left[w_{4} h_{1}^{\perp g} h_{1}^{\perp g}\right] \cos (4 \phi)+\mathcal{O}\left(\frac{q_{T}}{Q}\right)
\end{aligned}
$$

where the $F_{i}$ factors consist of specific combinations of $g g \rightarrow X_{0,2} \rightarrow \gamma \gamma$ helicity amplitudes, with $F_{3,4}$ involving amplitudes with opposite gluon helicities. The convolution $\mathcal{C}$ is defined as

$$
\begin{array}{r}
\mathcal{C}[w f g] \equiv \int \mathrm{d}^{2} \boldsymbol{p}_{T} \int \mathrm{d}^{2} \boldsymbol{k}_{T} \delta^{2}\left(\boldsymbol{p}_{T}+\boldsymbol{k}_{T}-\boldsymbol{q}_{T}\right) \\
w\left(\boldsymbol{p}_{T}, \boldsymbol{k}_{T}\right) f\left(x_{1}, \boldsymbol{p}_{T}^{2}\right) g\left(x_{2}, \boldsymbol{k}_{T}^{2}\right)
\end{array}
$$

and the weights appearing in the convolutions as

$$
\begin{aligned}
w_{2} & \equiv \frac{2\left(\boldsymbol{k}_{T} \cdot \boldsymbol{p}_{T}\right)^{2}-\boldsymbol{k}_{T}^{2} \boldsymbol{p}_{T}^{2}}{4 M_{p}^{4}}, \\
w_{3} & \equiv \frac{\boldsymbol{q}_{T}^{2} \boldsymbol{k}_{T}^{2}-2\left(\boldsymbol{q}_{T} \cdot \boldsymbol{k}_{T}\right)^{2}}{2 M_{p}^{2} \boldsymbol{q}_{T}^{2}}, \\
w_{4} & \equiv 2\left[\frac{\boldsymbol{p}_{T} \cdot \boldsymbol{k}_{T}}{2 M_{p}^{2}}-\frac{\left(\boldsymbol{p}_{T} \cdot \boldsymbol{q}_{T}\right)\left(\boldsymbol{k}_{T} \cdot \boldsymbol{q}_{T}\right)}{M_{p}^{2} \boldsymbol{q}_{T}^{2}}\right]^{2}-\frac{\boldsymbol{p}_{T}^{2} \boldsymbol{k}_{T}^{2}}{4 M_{p}^{4}} .
\end{aligned}
$$

The TMD distribution functions contain both perturbative and non-perturbative information. The tails $\left(\boldsymbol{p}_{T} \gg M_{p}\right)$ of the distribution functions can be calculated using pQCD, but the low $\boldsymbol{p}_{T}$ region will inevitably contain non-perturbative hadronic information. To get a description over the full $\boldsymbol{p}_{T}$ range one needs to extract the TMD distribution functions from experimental data $[22,23]$.

To make numerical predictions we will use a functional form for the unpolarized gluon TMD which has, in accordance with the pQCD calculation, a $1 / \boldsymbol{p}_{T}^{2}$ tail at large $\boldsymbol{p}_{T}$ and resembles a Gaussian for small $\boldsymbol{p}_{T}$,

$f_{1}^{g}\left(x, \boldsymbol{p}_{T}^{2}, \frac{3}{2} \sqrt{s}, M_{h}\right)=\frac{A_{0} M_{0}^{2}}{M_{0}^{2}+\boldsymbol{p}_{T}^{2}} \exp \left[-\frac{\boldsymbol{p}_{T}^{2}}{a \boldsymbol{p}_{T}^{2}+2 \sigma^{2}}\right]$.

Preferably one would fit the parameters in Eq. (6) to actual data, but since those are currently not available we will instead fit to the Standard Model Higgs boson transverse momentum distribution obtained by interfacing the POWHEG [24-26] NLO gluon fusion calculation [27] to Pythia 8.170 [28, 29], assuming a Higgs mass of $125 \mathrm{GeV}$ and a collider center of mass energy of $8 \mathrm{TeV}$. Pythia does not take into account effects of gluon polarization, so we fit the data by setting the linearly polarized gluon distribution equal to zero. In this way the TMD prediction without gluon polarization agrees with the Pythia prediction. We think this is the most realistic choice we can make, because Pythia is tuned to reproduce collider data well. Our Gaussian-with-tail Ansatz is able to adequately fit the Pythia data, as is shown in Figure 1. The fit results in the following values for the parameters $\sigma=38.9 \mathrm{GeV}, a=0.555$ and $M_{0}=3.90 \mathrm{GeV}$. We are not concerned about the overall normalization, as we will be only interested in distributions and not the absolute size of the cross section.

The linearly polarized gluon distribution will be expressed in terms of the unpolarized gluon distribution and the degree of polarization $\mathcal{P}$, i.e.,

$h_{1}^{\perp g}\left(x, \boldsymbol{p}_{T}, \zeta, \mu\right)=\mathcal{P}\left(x, \boldsymbol{p}_{T}^{2}, \zeta\right) \frac{2 M_{p}^{2}}{\boldsymbol{p}_{T}^{2}} f_{1}^{g}\left(x, \boldsymbol{p}_{T}, \zeta, \mu\right)$, 


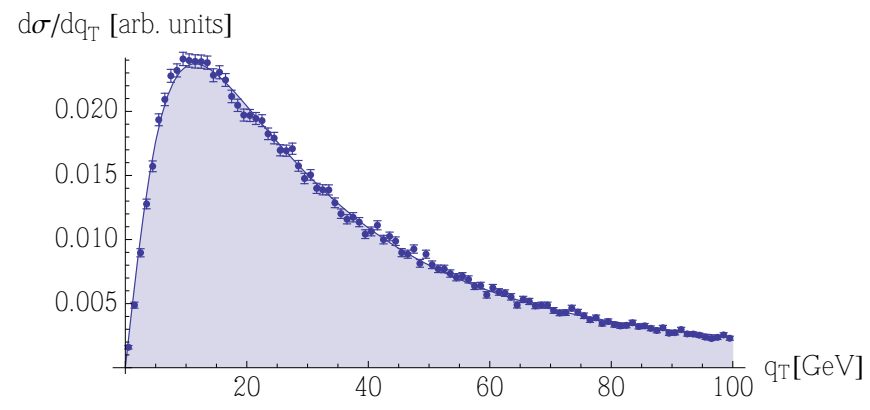

FIG. 1. Plot of $q_{T} \mathcal{C}\left[f_{1}^{g} f_{1}^{g}\right]$ (line) and the Pythia Higgs $\mathrm{d} \sigma / \mathrm{d} q_{T}$ distribution for $M_{h}=125 \mathrm{GeV}$ at $\sqrt{s}=8 \mathrm{TeV}$ (points).

such that $|\mathcal{P}|=1$ corresponds to $h_{1}^{\perp g}$ saturating its upper bound [30] and with the correct power law tail as first calculated in [19]. Calculations of the gluon TMD distributions using the Color Glass Condensate model predict maximal gluon polarization for large $\boldsymbol{p}_{T}$ and small $x$ [31]. Ideally one extracts the degree of polarization from data, but this is currently unfeasible.

Perturbative QCD can be used to calculate the large $\boldsymbol{p}_{T}$ tails of the TMD distributions in terms of the collinear parton distribution functions as has been done in Ref. [21] for the unpolarized distribution and Ref. [19] for the linearly polarized gluon distribution. We will follow a similar approach, but keep finite $\zeta$ instead of taking the $\zeta \rightarrow \infty$ limit and calculate the degree of polarization to leading order in $\alpha_{s}$ from the MSTW 2008 collinear parton distributions [32] evaluated at a scale of $\mu=2$ $\mathrm{GeV}$.

The pQCD calculation is only valid in the limit $\boldsymbol{p}_{T} \gg$ $M_{p}$. To model the lack of knowledge at low $\boldsymbol{p}_{T}$, we will define three different degrees of polarization $\mathcal{P}_{\text {min }}, \mathcal{P}$ and $\mathcal{P}_{\text {max }}$, of which the first approaches zero at low $\boldsymbol{p}_{T}$, the second follows the pQCD prediction and the last reaches up to one at low $\boldsymbol{p}_{T}$. Other sources of uncertainty are the choices of the scales $\zeta$ and $\mu$ and the omission of higher order terms. We estimate this additional uncertainty, by varying the different scales, to be maximally $10 \%$ and model it by letting $\mathcal{P}_{\max , \min }$ approach the pQCD calculation $\pm 10 \%$ for large $\boldsymbol{p}_{T}$. More specifically, we define

$$
\begin{aligned}
\mathcal{P}_{\text {min }} & \equiv \frac{\boldsymbol{p}_{T}^{4}}{p_{0}^{4}+\boldsymbol{p}_{T}^{4}} 0.9 \mathcal{P}_{\mathrm{pQCD}}\left(x, \boldsymbol{p}_{T}^{2}\right), \\
\mathcal{P} & \equiv \mathcal{P}_{\mathrm{pQCD}}\left(x, \boldsymbol{p}_{T}^{2}\right), \\
\mathcal{P}_{\text {max }} & \equiv 1-\frac{\boldsymbol{p}_{T}^{4}}{p_{0}^{4}+\boldsymbol{p}_{T}^{4}}\left[1-1.1 \mathcal{P}_{\mathrm{pQCD}}\left(x, \boldsymbol{p}_{T}^{2}\right)\right],
\end{aligned}
$$

where $\mathcal{P}_{\mathrm{pQCD}}$ is the pQCD degree of polarization calculated at $\zeta=1.5 \sqrt{s}$ and we take $p_{0}=5 \mathrm{GeV}$. The resulting $\mathcal{P}_{\text {min }}, \mathcal{P}$ and $\mathcal{P}_{\text {max }}$ are plotted in Figure 2.

We will consider the partonic process $g g \rightarrow X_{0,2} \rightarrow \gamma \gamma$ where $X$ is either a spin-0 or spin- 2 boson, with completely general couplings. For the interaction vertex we will follow the conventions of Refs. [11] and [12], where

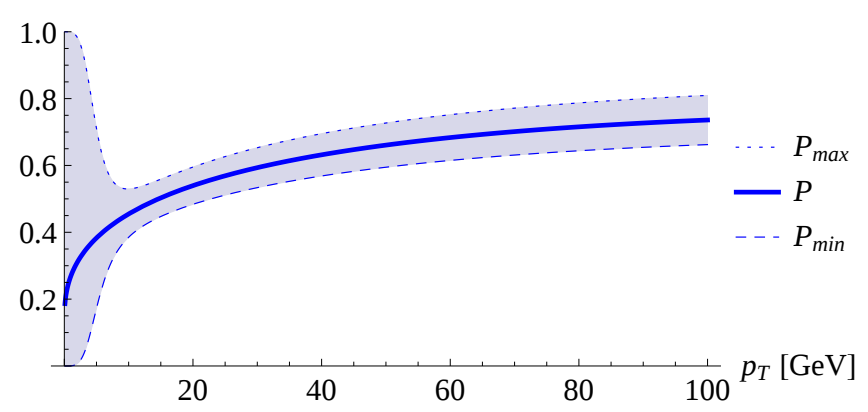

FIG. 2. Plot of the degrees of polarization $\mathcal{P}_{\text {min }}, \mathcal{P}$ and $\mathcal{P}_{\text {max }}$ at $x=M_{h} / \sqrt{s}$, with $M_{h}=125 \mathrm{GeV}$ and $\sqrt{s}=8 \mathrm{TeV}$.

the vertex coupling a spin-0 boson to massless gauge bosons is parameterized as

$V\left[X_{0} \rightarrow V^{\mu}\left(q_{1}\right) V^{\nu}\left(q_{2}\right)\right]=a_{1} q^{2} g^{\mu \nu}+a_{3} \epsilon^{q_{1} q_{2} \mu \nu}$,

and for a spin-2 boson as

$$
\begin{aligned}
V\left[X_{2}^{\alpha \beta} \rightarrow V^{\mu}\left(q_{1}\right) V^{\nu}\left(q_{2}\right)\right] & =\frac{1}{2} c_{1} q^{2} g^{\mu \alpha} g^{\nu \beta} \\
& +\left(c_{2} q^{2} g^{\mu \nu}+c_{5} \epsilon^{q_{1} q_{2} \mu \nu}\right) \frac{\tilde{q}^{\alpha} \tilde{q}^{\beta}}{q^{2}},
\end{aligned}
$$

where $q \equiv q_{1}+q_{2}$ and $\tilde{q} \equiv q_{1}-q_{2}$. The coupling to gluons can be different from the coupling to photons, but to keep expressions compact we will consider them equal.

For the $g g \rightarrow X_{0} \rightarrow \gamma \gamma$ subprocess, the non-zero $F$ factors in Eq. (3) read

$$
\begin{aligned}
& F_{1}=16\left|a_{1}\right|^{4}+8\left|a_{1}\right|^{2}\left|a_{3}\right|^{2}+\left|a_{3}\right|^{2}, \\
& F_{2}=16\left|a_{1}\right|^{4}-\left|a_{3}\right|^{4},
\end{aligned}
$$

and for the $g g \rightarrow X_{2} \rightarrow \gamma \gamma$ process one has

$$
\begin{aligned}
F_{1}= & 18 A^{+}\left|c_{1}\right|^{2} s_{\theta}^{4}+A^{+^{2}}\left(1-3 c_{\theta}^{2}\right)^{2} \\
& +\frac{9}{8}\left|c_{1}\right|^{4}\left(28 c_{2 \theta}+c_{4 \theta}+35\right), \\
F_{2}= & 9 A^{-}\left|c_{1}\right|^{2} s_{\theta}^{4}+A^{-} A^{+}\left(1-3 c_{\theta}^{2}\right)^{2}, \\
F_{3}= & 3 s_{\theta}^{2} B^{-}\left[3\left|c_{1}\right|^{2}\left(c_{2 \theta}+3\right)+A^{+}\left(3 c_{2 \theta}+1\right)\right], \\
F_{3}^{\prime}= & 6 s_{\theta}^{2} \operatorname{Re}\left(c_{1} c_{5}^{*}\right)\left[3\left|c_{1}\right|^{2}\left(c_{2 \theta}+3\right)+A^{+}\left(3 c_{2 \theta}+1\right)\right], \\
F_{4}= & 9 s_{\theta}^{4}\left|c_{1}\right|^{2}\left[2 B^{+}+4\left|c_{5}\right|^{2}\right],
\end{aligned}
$$

where we have defined $A^{ \pm} \equiv\left|c_{1}+4 c_{2}\right|^{2} \pm 4\left|c_{5}\right|^{2}, B^{ \pm} \equiv$ $\left|c_{1}+2 c_{2}\right|^{2} \pm 4\left|c_{2}\right|^{2}, c_{n \theta} \equiv \cos (n \theta)$ and $s_{\theta} \equiv \sin (\theta)$. Overall factors have been dropped, because as said we will be only interested in distributions and not the absolute size of the cross section. Unlike the case for Higgs production from linearly polarized photons [33], there is no direct observable signalling $C P$ violation in the spin- 0 case. For the spin- 2 case there is such a clear signature, being a $\sin 2 \phi$ dependence of the cross section, which can only 


\begin{tabular}{cccccccc} 
scenario & $0^{+}$ & $0^{-}$ & $2_{m}^{+}$ & $2_{h}^{+}$ & $2_{h^{\prime}}^{+}$ & $2_{h^{\prime \prime}}^{+}$ & $2_{h}^{-}$ \\
\hline \hline$a_{1}$ & 1 & 0 & - & - & - & - & - \\
$a_{3}$ & 0 & 1 & - & - & - & - & - \\
$c_{1}$ & - & - & 1 & 0 & 1 & 1 & 0 \\
$c_{2}$ & - & - & $-\frac{1}{4}$ & 1 & 1 & $-\frac{3}{2}$ & 0 \\
$c_{5}$ & - & - & 0 & 0 & 0 & 0 & 1
\end{tabular}

TABLE I. Different spin, parity and coupling scenarios.

be present if both $c_{1}$ and $c_{5}$ are non-zero, implying a $C P$-violating interaction.

In Ref. [12] a set of different spin, parity and coupling scenarios is defined. To those scenarios we will add $2_{h^{\prime}}^{+}$and $2_{h^{\prime \prime}}^{+}$, which will serve as examples of higherdimensional spin-2 coupling hypotheses that are indistinguishable in the $\theta$ distribution, but do have a different $\phi$ distribution. The scenarios are summarized in Table I.

In Figure 3 we show the diphoton $\cos \theta$ distribution for the various scenarios. Looking only at this distribution $0^{+}$and $0^{-}$are indistinguishable, as are $2_{h}^{+}$and $2_{h}^{-}$, and also $2_{h^{\prime}}^{+}$and $2_{h^{\prime \prime}}^{+}$.

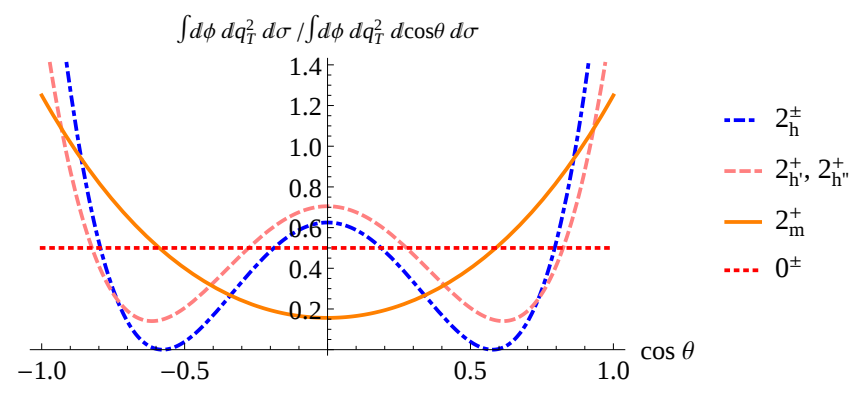

FIG. 3. Plot of the $\cos \theta$ distribution for the various scenarios.

In Figure 4 we show the diphoton transverse momentum distribution for the different coupling hypotheses at fixed $\theta=\pi / 2$ and at zero rapidity. The positive parity states show an enhancement at low $q_{T}(<15 \mathrm{GeV})$ with respect to the negative parity states. At high $q_{T}(>15$ $\mathrm{GeV}$ ) this is reversed, but with such a strongly reduced magnitude that it is invisible in the plot. The $q_{T}$ distribution can thus, in principle, be used to determine the parity of the newly found boson $[14,15]$. Although the difference is small and most likely difficult to measure experimentally, this is the only way we know to determine the parity in the $g g \rightarrow X_{0,2} \rightarrow \gamma \gamma$ channel.

Figure 5 shows the diphoton $\phi$ distribution for the selected scenarios at fixed $\theta=\pi / 2$ and at zero rapidity. The scalar, pseudoscalar and $2_{h}^{ \pm}$hypotheses show a uniform $\phi$ distribution, whereas the $2_{m}^{+}$has a characteristic $\cos (4 \phi)$ dependence with an amplitude of $5.4_{-1.8}^{+3.7} \%$. The $2_{h^{\prime}}^{+}$and $2_{h^{\prime \prime}}^{+}$scenarios exhibit a weak $\cos (4 \phi)$ modulation with an amplitude of $1.2_{-0.4}^{+0.8 \%}$ and a strong $\cos (2 \phi)$ modulation with an amplitude of $24 \pm 3 \%$ and opposite sign. The $\phi$ distribution thus offers a way to distinguish

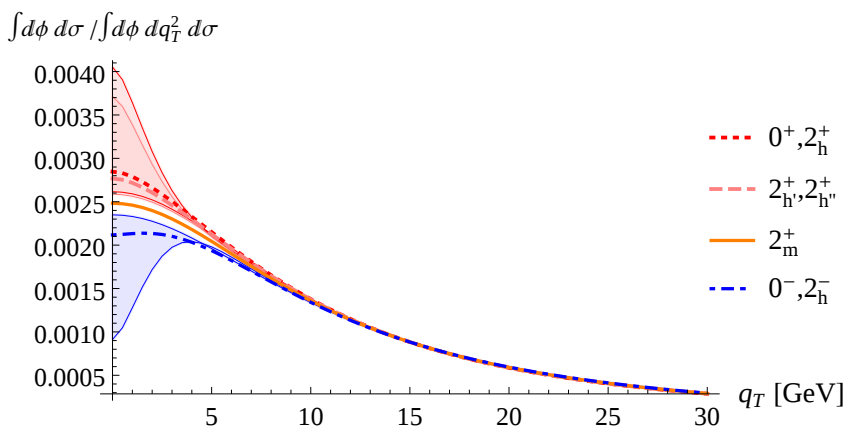

FIG. 4. Plot of the $q_{T}$ distribution for the various coupling schemes at $\theta=\pi / 2$ and zero rapidity, using an upper limit on the $q_{T}$ integration in the denominator of $M_{h} / 2$. The shaded area is due to the uncertainty in the degree of polarization.

$0^{ \pm}, 2_{m}^{+}, 2_{h^{\prime}}^{+}$and $2_{h^{\prime \prime}}^{+}$from each other, something that is impossible with the $\cos \theta$ distribution alone.

We want to stress again that a $\sin 2 \phi$ dependence implies a $C P$-violation coupling, which is thus very interesting to search for. Note however that Higgs bosons produced with positive and negative rapidity have to be treated separately, because those regions will have an opposite sign $\sin 2 \phi$ modulation and would otherwise cancel. We also want to mention that $g g \rightarrow \gamma \gamma$ continuum production has a non-isotropic $\phi$ dependence, with an amplitude approximately a factor 3 smaller than resonance production $[22,34]$, which should not be mistaken for a spin-2 Higgs.

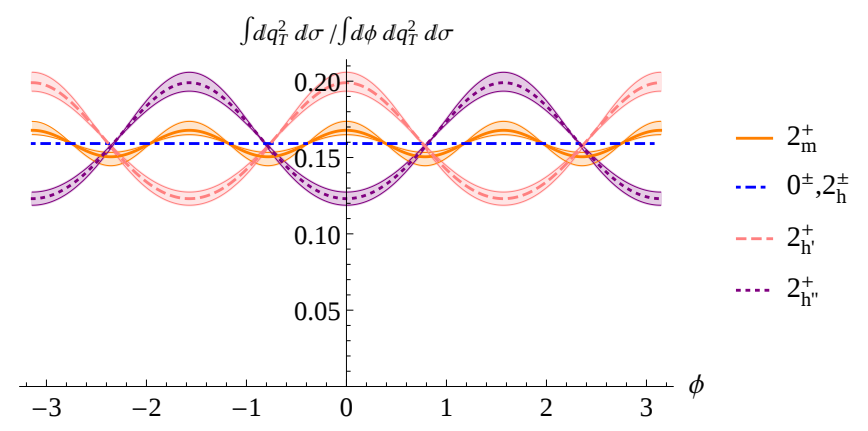

FIG. 5. Plot of the $\phi$ distribution for the different benchmark scenarios at $\theta=\pi / 2$ and zero rapidity, using an upper limit on the $q_{T}$ integration of $M_{h} / 2$. The shaded area is due to the uncertainty in the degree of polarization.

In conclusion, we have calculated the diphoton distribution in the decay of arbitrary spin-0 and spin- 2 bosons produced from gluon fusion, taking into account the fact that gluons inside an unpolarized proton are generally linearly polarized. The gluon polarization brings about a difference in the transverse momentum distribution of positive and negative parity states. At the same time, it causes the azimuthal CS angle $\phi$ distribution to be nonisotropic for various spin-2 coupling hypotheses. These distributions allow spin and parity scenarios to be dis- 
tinguished that cannot be done with the polar angle $\theta$ dependence alone. We think that these observables could therefore form a valuable addition to the analysis methods to determine the spin, parity and coupling of the newly found boson at the LHC.

This work was supported in part by the German Bundesministerium für Bildung und Forschung (BMBF), grant no. 05P12VTCTG.

[1] G. Aad et al. [ATLAS Collaboration], Phys. Lett. B 716, 1 (2012) [arXiv:1207.7214 [hep-ex]].

[2] S. Chatrchyan et al. [CMS Collaboration], Phys. Lett. B 716, 30 (2012) [arXiv:1207.7235 [hep-ex]].

[3] http://cds.cern.ch/record/1527124/files/ ATLAS-CONF-2013-029.pdf

[4] http://cds.cern.ch/record/1523699/files/ ATLAS-CONF-2013-013.pdf

[5] http://cds.cern.ch/record/1527127/files/ ATLAS-CONF-2013-031.pdf

[6] http://cds.cern.ch/record/1523767/files/ HIG-13-002-pas.pdf

[7] http://cds.cern.ch/record/1523673/files/ HIG-13-003-pas.pdf

[8] L. D. Landau, Dokl. Akad. Nauk Ser. Fiz. 60, 207 (1948).

[9] C. -N. Yang, Phys. Rev. 77, 242 (1950).

[10] J. Ellis, R. Fok, D. S. Hwang, V. Sanz and T. You, arXiv:1210.5229 [hep-ph].

[11] Y. Gao, A. V. Gritsan, Z. Guo, K. Melnikov, M. Schulze and N. V. Tran, Phys. Rev. D 81, 075022 (2010) [arXiv:1001.3396 [hep-ph]].

[12] S. Bolognesi, Y. Gao, A. V. Gritsan, K. Melnikov, M. Schulze, N. V. Tran and A. Whitbeck, Phys. Rev. D 86, 095031 (2012) [arXiv:1208.4018 [hep-ph]].

[13] J. C. Collins and D. E. Soper, Phys. Rev. D 16, 2219 (1977).

[14] D. Boer, W. J. den Dunnen, C. Pisano, M. Schlegel and W. Vogelsang, Phys. Rev. Lett. 108, 032002 (2012) [arXiv:1109.1444 [hep-ph]].

[15] W. J. den Dunnen, D. Boer, C. Pisano, M. Schlegel and
W. Vogelsang, arXiv:1205.6931 [hep-ph].

[16] K. Hagiwara, Q. Li and K. Mawatari, JHEP 0907, 101 (2009) [arXiv:0905.4314 [hep-ph]].

[17] J. R. Andersen, K. Arnold and D. Zeppenfeld, JHEP 1006, 091 (2010) [arXiv:1001.3822 [hep-ph]].

[18] J. Frank, M. Rauch and D. Zeppenfeld, arXiv:1211.3658 [hep-ph].

[19] P. Sun, B. -W. Xiao and F. Yuan, Phys. Rev. D 84, 094005 (2011) [arXiv:1109.1354 [hep-ph]].

[20] J. P. Ma, J. X. Wang and S. Zhao, arXiv:1211.7144 [hep$\mathrm{ph}$.

[21] X. -d. Ji, J. -P. Ma and F. Yuan, JHEP 0507, 020 (2005) [hep-ph/0503015].

[22] J. -W. Qiu, M. Schlegel and W. Vogelsang, Phys. Rev. Lett. 107, 062001 (2011) [arXiv:1103.3861 [hep-ph]].

[23] D. Boer, S. J. Brodsky, P. J. Mulders and C. Pisano, Phys. Rev. Lett. 106, 132001 (2011) [arXiv:1011.4225 [hep-ph]].

[24] S. Alioli, P. Nason, C. Oleari and E. Re, JHEP 0904, 002 (2009) [arXiv:0812.0578 [hep-ph]].

[25] P. Nason, JHEP 0411, 040 (2004) [hep-ph/0409146].

[26] S. Frixione, P. Nason and C. Oleari, JHEP 0711, 070 (2007) [arXiv:0709.2092 [hep-ph]].

[27] S. Alioli, P. Nason, C. Oleari and E. Re, JHEP 1006, 043 (2010) [arXiv:1002.2581 [hep-ph]].

[28] T. Sjostrand, S. Mrenna and P. Z. Skands, JHEP 0605, 026 (2006) [hep-ph/0603175].

[29] T. Sjostrand, S. Mrenna and P. Z. Skands, Comput. Phys. Commun. 178, 852 (2008) [arXiv:0710.3820 [hep-ph]].

[30] P. J. Mulders and J. Rodrigues, Phys. Rev. D 63, 094021 (2001) [hep-ph/0009343].

[31] A. Metz and J. Zhou, Phys. Rev. D 84, 051503 (2011) [arXiv:1105.1991 [hep-ph]].

[32] A. D. Martin, W. J. Stirling, R. S. Thorne and G. Watt, Eur. Phys. J. C 63, 189 (2009) [arXiv:0901.0002 [hep$\mathrm{ph}]$.

[33] B. Grzadkowski and J. F. Gunion, Phys. Lett. B 294, 361 (1992) [hep-ph/9206262].

[34] W. J. den Dunnen, "Polarization effects in proton-proton collisions within the Standard Model and beyond," (PhD Thesis) (2012) http://dare.ubvu.vu.nl/handle/1871/ 39659 